\title{
REAL HYPERSURFACES WITH CYCLIC-PARALLEL STRUCTURE JACOBI OPERATORS IN A NONFLAT COMPLEX SPACE FORM
}

\author{
U-HANG KI and HIROYUKI KURIHARA ${ }^{凶}$
}

(Received 22 May 2009)

\begin{abstract}
It is known that there are no real hypersurfaces with parallel structure Jacobi operators in a nonflat complex space form. In this paper, we classify real hypersurfaces in a nonflat complex space form whose structure Jacobi operator is cyclic-parallel.
\end{abstract}

2000 Mathematics subject classification: primary 53B25; secondary 53C15, 53C25.

Keywords and phrases: complex space form, real hypersurface, structure Jacobi operator, cyclic-parallel.

\section{Introduction}

A complex $n$-dimensional Kähler manifold with Kähler structure $J$ of constant holomorphic sectional curvature $4 c$ is called a complex space form and denoted by $M_{n}(c)$. As is well known, a connected complete and simply connected complex space form is complex analytically isometric to a complex projective space $P_{n} \mathbb{C}$, a complex Euclidean space $\mathbb{C}$ or a complex hyperbolic space $H_{n} \mathbb{C}$ if $c>0, c=0$ or $c<0$, respectively.

The study of real hypersurfaces in complex projective space $P_{n} \mathbb{C}$ was initiated by Takagi [14], who proved that all homogeneous real hypersurfaces in $P_{n} \mathbb{C}$ could be divided into six types which are said to be of type $A_{1}, A_{2}, B, C, D$ and $E$. He showed also in [15] and [16] that if a real hypersurface $M$ in $P_{n} \mathbb{C}$ has two or three distinct constant principal curvatures, then $M$ is locally congruent to one of the homogeneous ones of type $A_{1}, A_{2}$ or $B$. In particular, real hypersurfaces of type $A_{1}, A_{2}$ and $B$ in $P_{n} \mathbb{C}$ have been studied by several authors (see Cecil and Ryan [3], Maeda [8] and Okumura [11]).

In the case of complex hyperbolic space $H_{n} \mathbb{C}$, Montiel and Romero started the study of real hypersurfaces in [9] and constructed some homogeneous real hypersurfaces in $H_{n} \mathbb{C}$ which are said to be of type $A_{0}, A_{1}$ and $A_{2}$. Those hypersurfaces have a lot of nice geometric properties (see Berndt [1] and Montiel and Romero [9]). In 2007 Berndt and Tamaru [2] classified all homogeneous real hypersurfaces in $H_{n} \mathbb{C}$.

(C) 2009 Australian Mathematical Publishing Association Inc. 0004-9727/2009 \$16.00 
Real hypersurfaces of each type in $M_{n}(c)$ have been described in detail by Niebergall and Ryan [10].

On the other hand, the Jacobi operator field with respect to $X$ in a Riemannian manifold $M$ is defined by $R_{X}=R(\cdot, X) X$, where $R$ denotes the Riemannian curvature tensor of $M$. Let $M$ be a real hypersurface in $M_{n}(c), c \neq 0$, and $v$ a unit normal vector field on $M$. Then a tangent vector field $\xi:=-J v$ to $M$ is called the structure vector field on $M$. We will call the Jacobi operator on $M$ with respect to $\xi$ the structure Jacobi operator on $M$. The structure Jacobi operator $R \xi=R(\cdot, \xi) \xi$ has a fundamental role in contact geometry. Cho and the first author started the study of real hypersurfaces in a complex space form by using the operator $R_{\xi}$ (see [4]). Recently Ortega et al. [12] proved the nonexistence of real hypersurfaces in nonflat complex space forms with parallel structure Jacobi operator. More generally, such a result has been extended by [13] due to them.

Now let $M$ be a real hypersurfaces in a complex space form $M_{n}(c), c \neq 0$. The structure Jacobi operator $R_{\xi}$ of $M$ is said to be cyclic-parallel if it satisfies

$$
\mathfrak{S} R_{\xi}^{\prime}(X, Y, Z)=\mathfrak{S} g\left(\nabla_{X} R_{\xi}(Y), Z\right)=0
$$

for any vector fields $X, Y$ and $Z$, where $\mathfrak{S}$ and $\nabla$ denote the cyclic sum and the Riemannian connection, respectively. In Section 5, the structure Jacobi operator of real hypersurfaces in $P_{n} \mathbb{C}$ of type $A_{1}, A_{2}$ and a special case of type $B$, and $H_{n} \mathbb{C}$ of type $A_{0}, A_{1}$ and $A_{2}$ are cyclic-parallel. The purpose of this paper is to investigate this converse problem.

THEOREM 1.1. Let $M$ be a real hypersurface in a complex space form $M_{n}(c), c \neq 0$, $n \geq 3$. If the structure Jacobi operator is cyclic-parallel, then $M$ is locally congruent to one of the following.

(I) In the case where $M_{n}(c)=P_{n} \mathbb{C}$ :

$\left(A_{1}\right)$ a geodesic hypersphere of radius $r$, where $0<r<\pi / \sqrt{4 c}$;

$\left(A_{2}\right)$ a tube of radius $r$ over a totally geodesic $P_{k} \mathbb{C}$ for some $k \in\{1, \ldots, n-2\}$, where $0<r<\pi / \sqrt{4 c}$;

(B) a tube of radius $r$ over complex quadric $Q_{n-1}$, where $\cot r=(\sqrt{2 c+4}+$ $\sqrt{2 c}) / 2$.

(II) In the case where $M_{n}(c)=H_{n} \mathbb{C}$ :

$\left(A_{0}\right)$ a horosphere;

$\left(A_{1}\right)$ a geodesic hypersphere or a tube over a complex hyperbolic hyperplane $H_{n-1} \mathbb{C}$;

$\left(A_{2}\right)$ a tube over a totally geodesic $H_{k} \mathbb{C}$ for some $k \in\{1, \ldots, n-2\}$.

All manifolds in this paper are assumed to be connected and of class $C^{\infty}$ and the real hypersurfaces are supposed to be oriented.

\section{Preliminaries}

We denote by $M_{n}(c), c \neq 0$, a nonflat complex space form with the Fubini-Study metric $\tilde{g}$ of constant holomorphic sectional curvature $4 c$ and Levi-Civita connection $\tilde{\nabla}$. 
For an immersed $(2 n-1)$-dimensional Riemannian manifold $\tau: M \rightarrow M_{n}(c)$, the Levi-Civita connection $\nabla$ of induced metric and the shape operator $H$ of the immersion are characterized by

$$
\tilde{\nabla}_{X} Y=\nabla_{X} Y+g(H X, Y) v, \quad \tilde{\nabla}_{X} v=-H X,
$$

for any vector fields $X$ and $Y$ on $M$, where $g$ denotes the Riemannian metric of $M$ induced from $\tilde{g}$ and $v$ a unit normal vector on $M$. In the sequel the indices $i, j, k, l, \ldots$ run over the range $\{1,2, \ldots, 2 n-1\}$ unless otherwise stated. For a local orthonormal frame field $\left\{e_{i}\right\}$ of $M$, we denote the dual 1-forms by $\left\{\theta_{i}\right\}$. Then the connection forms $\theta_{i j}$ are defined by

$$
d \theta_{i}+\sum_{j} \theta_{i j} \wedge \theta_{j}=0, \quad \theta_{i j}+\theta_{j i}=0 .
$$

Then

$$
\nabla_{e_{i}} e_{j}=\sum_{k} \theta_{k j}\left(e_{i}\right) e_{k}=\sum_{k} \Gamma_{k i j} e_{k},
$$

where we put $\theta_{i j}=\sum_{k} \Gamma_{i j k} \theta_{k}$. The almost contact metric structure $\left(\phi=\left(\phi_{i j}\right)\right.$, $\left.\xi=\sum_{i} \xi_{i} e_{i}\right)$ is induced on $M$ by the equation

$$
J\left(e_{i}\right)=\sum_{j} \phi_{j i} e_{j}+\xi_{i} v
$$

Then the structure tensor $\phi$ and the structure vector $\xi$ satisfy

$$
\begin{gathered}
\sum_{k} \phi_{i k} \phi_{k j}=\xi_{i} \xi_{j}-\delta_{i j}, \quad \sum_{j} \xi_{j} \phi_{i j}=0, \quad \sum_{i} \xi_{i}^{2}=1, \quad \phi_{i j}+\phi_{j i}=0, \\
d \phi_{i j}=\sum_{k}\left(\phi_{i k} \theta_{k j}-\phi_{j k} \theta_{k i}-\xi_{i} h_{j k} \theta_{k}+\xi_{j} h_{i k} \theta_{k}\right), \\
d \xi_{i}=\sum_{j} \xi_{j} \theta_{j i}-\sum_{j, k} \phi_{j i} h_{j k} \theta_{k} .
\end{gathered}
$$

We denote the components of the shape operator or the second fundamental tensor $H$ of $M$ by $h_{i j}$. The components $h_{i j ; k}$ of the covariant derivative of $H$ are given by $\sum_{k} h_{i j ; k} \theta_{k}=d h_{i j}-\sum_{k} h_{i k} \theta_{k j}-\sum_{k} h_{j k} \theta_{k i}$. Then we have the equations of Gauss and Codazzi,

$$
\begin{gathered}
R_{i j k l}=c\left(\delta_{i k} \delta_{j l}-\delta_{i l} \delta_{j k}+\phi_{i k} \phi_{j l}-\phi_{i l} \phi_{j k}+2 \phi_{i j} \phi_{k l}\right)+h_{i k} h_{j l}-h_{i l} h_{j k}, \\
h_{i j ; k}-h_{i k ; j}=c\left(\xi_{k} \phi_{i j}-\xi_{j} \phi_{i k}+2 \xi_{i} \phi_{k j}\right)
\end{gathered}
$$

respectively.

From (2.2) the structure Jacobi operator $R_{\xi}=\left(\Xi_{i j}\right)$ is given by

$$
\Xi_{i j}=\sum_{k, l} h_{i k} h_{j l} \xi_{k} \xi_{l}-\sum_{k, l} h_{i j} h_{k l} \xi_{k} \xi_{l}+c \xi_{i} \xi_{j}-c \delta_{i j} .
$$


First, we give the following lemma.

LEMMA 2.1. Let $U$ be an open set in $M$ and $F$ a smooth function on $U$. We put $d F=\sum_{i} F_{i} \theta_{i}$. Then

$$
F_{i j}-F_{j i}=\sum_{k} F_{k} \Gamma_{k i j}-\sum_{k} F_{k} \Gamma_{k j i}
$$

PROOF. Taking the exterior derivative of $d F=\sum_{i} F_{i} \theta_{i}$ immediately gives the formula .

REMARK 2.2. We have already proved [5, Lemma 2.1].

Now we take a local orthonormal frame field $e_{i}$ in such a way that:

(1) $e_{1}=\xi$;

(2) $e_{2}$ is in the direction of $\sum_{i=2}^{2 n-1} h_{1 i} e_{i}$; and

(3) $e_{3}=\phi e_{2}$.

Then

$$
\xi_{1}=1, \quad \xi_{i}=0(i \geq 2), \quad h_{1 j}=0(j \geq 3) \quad \text { and } \quad \phi_{32}=1 .
$$

We put $\alpha:=h_{11}, \beta:=h_{12}, \gamma:=h_{22}, \varepsilon:=h_{23}$ and $\delta:=h_{33}$.

Hereafter the indices $p, q, r, s, \ldots$ run over the range $\{4,5, \ldots, 2 n-1\}$ unless otherwise stated.

Since $d \xi_{i}=0$,

$$
\begin{aligned}
\theta_{12} & =\varepsilon \theta_{2}+\delta \theta_{3}+\sum_{p} h_{3 p} \theta_{p}, \\
\theta_{13} & =-\beta \theta_{1}-\gamma \theta_{2}-\varepsilon \theta_{3}-\sum_{p} h_{2 p} \theta_{p}, \\
\theta_{1 p} & =\sum_{q} \phi_{q p} h_{q 2} \theta_{2}+\sum_{q} \phi_{q p} h_{q 3} \theta_{3}+\sum_{q, r} \phi_{q p} h_{q r} \theta_{r} .
\end{aligned}
$$

We put

$$
\theta_{23}=\sum_{i} X_{i} \theta_{i}, \quad \theta_{2 p}=\sum_{i} Y_{p i} \theta_{i}, \quad \theta_{3 p}=\sum_{i} Z_{p i} \theta_{i} .
$$

Then it follows from $d \phi_{2 i}=0$ that $Y_{p i}=-\sum_{q} \phi_{p q} Z_{q i}$ or $Z_{p i}=\sum_{q} \phi_{p q} Y_{q i}$. Equation (2.4) is rewritten as

$$
\Xi_{i j}=-\alpha h_{i j}+h_{1 i} h_{1 j}+c \delta_{i 1} \delta_{j 1}-c \delta_{i j} .
$$

Some fundamental properties about the structure vector and the principal curvature are stated for later use.

Proposition 2.3 (Meada [8], Ki and Suh [6]). Let $M$ be a real hypersurface in $M_{n}(c), c \neq 0$. If the structure vector $\xi$ is principal, then the corresponding principal curvature $\alpha$ is locally constant.

PROPOSITION 2.4 (Niebergall and Ryan [10]). Let $M$ be a real hypersurface in $M_{n}(c), c \neq 0$. Assume that $\xi$ is a principal curvature vector and the corresponding principal curvature is $\alpha$. If $H X=r X$ for $X \perp \xi$, then $(2 r-\alpha) H \phi X=(\alpha r+2 c) \phi X$. 


\section{Real hypersurfaces with cyclic-parallel structure Jacobi operator}

First we assume that $\mathfrak{S} R_{\xi}^{\prime}(X, Y, Z)=0$ for any vector fields $X, Y$ and $Z$. The components $\Xi_{i j ; k}$ of the covariant derivative of $R_{\xi}=\left(\Xi_{i j}\right)$ are given by

$$
\sum_{k} \Xi_{i j ; k} \theta_{k}=d \Xi_{i j}-\sum_{k} \Xi_{k j} \theta_{k i}-\sum_{k} \Xi_{i k} \theta_{k j}
$$

Substituting (2.8) into the above equation,

$$
\begin{aligned}
\sum_{k} \Xi_{i j ; k} \theta_{k}=-( & d \alpha) h_{i j}-\alpha d h_{i j}+\left(d h_{1 i}\right) h_{1 j}+h_{1 i}\left(d h_{1 j}\right) \\
& +\alpha \sum_{k} h_{k j} \theta_{k i}-\alpha h_{1 j} \theta_{1 i}-\beta h_{1 j} \theta_{2 i}-c \delta_{j 1} \theta_{1 i} \\
& +\alpha \sum_{k} h_{i k} \theta_{k j}-\alpha h_{1 i} \theta_{1 j}-\beta h_{1 i} \theta_{2 j}-c \delta_{i 1} \theta_{1 j} .
\end{aligned}
$$

Our assumption $\mathfrak{S} R_{\xi}^{\prime}(X, Y, Z)=0$ for any vector fields $X, Y$ and $Z$ is equivalent to $\Xi_{i j ; k}+\Xi_{j k ; i}+\Xi_{k i ; j}=0$. This equation is rewritten as

$$
\begin{aligned}
& \alpha_{k} h_{i j}+\alpha_{i} h_{j k}+\alpha_{j} h_{k i}+\alpha\left(h_{i j k}+h_{j k i}+h_{k i j}\right) \\
& \quad-h_{1 j} h_{1 i k}-h_{1 k} h_{1 j i}-h_{1 i} h_{1 k j}-h_{1 j} h_{1 k i}-h_{1 k} h_{1 i j}-h_{1 i} h_{1 j k} \\
& \quad+\alpha h_{1 j}\left(\Gamma_{1 i k}+\Gamma_{1 k i}\right)+\alpha h_{1 k}\left(\Gamma_{1 j i}+\Gamma_{1 i j}\right)+\alpha h_{1 i}\left(\Gamma_{1 k j}+\Gamma_{1 j k}\right) \\
& \quad+\beta h_{1 j}\left(\Gamma_{2 i k}+\Gamma_{2 k i}\right)+\beta h_{1 k}\left(\Gamma_{2 j i}+\Gamma_{2 i j}\right)+\beta h_{1 i}\left(\Gamma_{2 k j}+\Gamma_{2 j k}\right) \\
& \quad+c \delta_{1 j}\left(\Gamma_{1 i k}+\Gamma_{1 k i}\right)+c \delta_{1 k}\left(\Gamma_{1 j i}+\Gamma_{1 i j}\right)+c \delta_{1 i}\left(\Gamma_{1 k j}+\Gamma_{1 j k}\right) \\
& \quad-\alpha \sum_{l} h_{l j}\left(\Gamma_{l i k}+\Gamma_{l k j}\right)-\alpha \sum_{l} h_{l k}\left(\Gamma_{l j i}+\Gamma_{l i j}\right) \\
& \quad-\alpha \sum_{l} h_{l i}\left(\Gamma_{l k j}+\Gamma_{l j k}\right)=0,
\end{aligned}
$$

because of (3.1).

In the following we assume that $\beta \neq 0$. Let $i, j, k \in\{1,2,3, p, q\}$. Then equation (3.2) can be stated as follows:

$$
\begin{aligned}
& \varepsilon=0, \quad h_{3 p}=0 \\
& \alpha \delta+c=0 \\
& \left(\beta^{2}-\alpha \gamma\right)_{1}-2 \alpha \sum_{p} h_{2 p} Y_{p 1}=0 \\
& (\alpha \gamma)_{3}+2\left(\beta^{2}-\alpha \gamma-c\right) X_{2}+2 \alpha \sum_{p} h_{2 p}\left(Y_{p 3}+Z_{p 2}\right)=0 \\
& \left(\beta^{2}-\alpha \gamma-c\right)\left(X_{1}-\delta\right)+\alpha \sum_{p} h_{2 p} Z_{p 1}=0 \\
& \left(\beta^{2}-\alpha \gamma\right)_{p}-2\left(\alpha h_{2 p}\right)_{2}-2\left(\beta^{2}-\alpha \gamma\right) Y_{p 2} \\
& \quad+2 \alpha \sum_{q} h_{2 q}\left(\Gamma_{q p 2}-Y_{q p}\right)=0 \\
& \quad
\end{aligned}
$$




$$
\begin{aligned}
& \left(\alpha h_{2 p}\right)_{1}+\alpha \sum_{q} h_{p q} Y_{q 1}+\left(\beta^{2}-\alpha \gamma\right) Y_{p 1}-\alpha \sum_{q} h_{2 q} \Gamma_{q p 1} \\
& \quad+\alpha \sum_{q, r} h_{2 q} \phi_{r q} h_{r p}=0, \\
& \alpha h_{2 p}\left(X_{1}-\delta\right)-\sum_{q}\left(\alpha h_{q p}+c \delta_{p q}\right) Z_{q 1}=0, \\
& \delta Z_{p 3}+h_{2 p} X_{3}=0, \\
& \left(\beta^{2}-\alpha \gamma-c\right) X_{3}+\alpha \sum_{p} h_{2 p} Z_{p 3}=0, \\
& \left(\alpha h_{2 p}\right)_{3}-\left(\beta^{2}-\alpha \gamma\right)\left(X_{p}+Y_{p 3}\right)+\alpha \delta\left(Z_{p 2}-X_{p}\right)+\alpha h_{2 p} X_{2} \\
& \quad+\alpha \sum_{r} h_{2 r}\left(\Gamma_{r p 3}-Z_{r p}\right)=0, \\
& \left(\alpha h_{p q}\right)_{1}-\alpha h_{2 q} Y_{p 1}-\alpha \sum_{r} h_{r q} \Gamma_{r p 1}-\alpha h_{2 p} Y_{q 1} \\
& \quad-\alpha \sum_{r} h_{p r} \Gamma_{r q 1}+c\left(\Gamma_{1 q p}-\Gamma_{1 p q}\right)=0 .
\end{aligned}
$$

Henceforth we shall use (3.3) without further mention.

Properly speaking, we should denote equation (2.3) by $(23)_{i j k}$, for example. In this paper we denote it simply by $(i j k)$. Then we have the following equations (112)$(q 3 p)$.

(112) $\alpha_{2}-\beta_{1}=0$,

(212) $\beta_{2}-\gamma_{1}-2 \sum_{p} h_{2 p} Y_{p 1}=0$,

(312) $(\alpha-\delta) \gamma-\beta X_{2}+(\gamma-\delta) X_{1}-\beta^{2}-\sum_{p} h_{2 p} Z_{p 1}=-c$,

(113) $\alpha_{3}+3 \beta \delta-\alpha \beta+\beta X_{1}=0$,

(213) $\beta_{3}-\alpha \delta+\gamma \delta+(\gamma-\delta) X_{1}-\beta^{2}-\sum_{p} h_{2 p} Z_{p 1}=c$,

(313) $\beta X_{3}+\delta_{1}=0$,

(223) $\gamma_{3}-2 \beta \delta+2 \sum_{p} h_{2 p} Y_{p 3}+(\gamma-\delta) X_{2}-\beta \gamma-\sum_{p} h_{2 p} Z_{p 2}=0$,

(323) $\sum_{p} h_{2 p} Z_{p 3}-\delta_{2}-(\gamma-\delta) X_{3}=0$,

(1p1) $\alpha_{p}+\beta Y_{p 1}=0$,

(12p) $\beta_{p}+2 \sum_{q, r} h_{2 q} \phi_{r q} h_{r p}+\beta Y_{p 2}+\alpha \sum_{q} \phi_{q p} h_{2 q}=0$,

(13p) $-2 \delta h_{2 p}+\beta Y_{p 3}+\alpha h_{2 p}-\beta X_{p}=0$, 
(22p) $\gamma_{p}+2 \sum_{q} h_{2 q} Y_{q p}-h_{2 p 2}-\sum_{q} h_{q p} Y_{q 2}$

$$
+\beta \sum_{q} \phi_{q p} h_{2 q}+\gamma Y_{p 2}+\sum_{q} h_{2 q} \Gamma_{q p 2}=0,
$$

(23p) $\delta X_{p}+\beta h_{2 p}-\gamma X_{p}+\sum_{q} h_{2 q} Z_{q p}-h_{2 p 3}$

$$
-\sum_{q} h_{q p} Y_{q 3}+\gamma Y_{p 3}+\sum_{q} h_{2 q} \Gamma_{q p 3}=0,
$$

(33p) $\delta_{p}+h_{2 p} X_{3}-\sum_{q} h_{q p} Z_{q 3}+\delta Z_{p 3}=0$,

(21p) $\beta_{p}+\sum_{q, r} h_{2 q} \phi_{r q} h_{r p}-h_{2 p 1}-\sum_{q} h_{q p} Y_{q 1}+\gamma Y_{p 1}+\sum_{q} h_{2 q} \Gamma_{q p 1}=0$,

(31p) $-\delta h_{2 p}+\alpha h_{2 p}-\beta X_{p}+h_{2 p} X_{1}-\sum_{q} h_{q p} Z_{q 1}+\delta Z_{p 1}=0$,

(32p) $\delta X_{p}+\beta h_{2 p}-\gamma X_{p}+\sum_{q} h_{2 q} Z_{q p}+h_{2 p} X_{2}-\sum_{q} h_{p q} Z_{q 2}+\delta Z_{p 2}=0$,

(2pq) $h_{2 p q}+\sum_{r} h_{r p} Y_{r q}-\beta \sum_{r} \phi_{r p} h_{r q}-\gamma Y_{p q}-\sum_{r} h_{2 r} \Gamma_{r p q}-h_{2 q p}$

$$
-\sum_{r} h_{r q} Y_{r p}+\beta \sum_{r} \phi_{r q} h_{r p}+\gamma Y_{q p}+\sum_{r} h_{2 r} \Gamma_{r q p}=0,
$$

(q1p) $\sum_{r, s} h_{r q} \phi_{s r} h_{s p}-\alpha \sum_{r} \phi_{r q} h_{r q}-\beta Y_{q p}-h_{p q 1}+h_{2 q} Y_{p 1}$

$$
+\sum_{r} h_{r q} \Gamma_{r p 1}+h_{2 p} Y_{q 1}+\sum_{r} h_{r p} \Gamma_{r q 1}=c \phi_{p q},
$$

$(q 3 p)-\delta Z_{q p}-h_{2 q} X_{p}+\sum_{r} h_{q r} Z_{r p}-h_{q p 3}+h_{q 2} Y_{p 3}$

$$
+\sum_{r} h_{q r} \Gamma_{r p 3}+h_{p 2} Y_{q 3}+\sum_{r} h_{p r} \Gamma_{r q 3}=0 .
$$

REMARK 3.1. We have omitted equations (1pq), $(3 p q),(p 2 q)$ and $(p q r)$ since we do not need them.

\section{Key lemma}

Suppose that $\beta \neq 0$. From (3.10) and (31p),

$$
\alpha h_{2 p}=\beta X_{p}
$$

This and (13p) imply that

$$
2 \delta h_{2 p}=\beta Y_{p 3},
$$

and so

$$
\sum_{p} h_{2 p} Z_{p 3}=0
$$


LEMma 4.1. $H\left(e_{2}\right) \in \operatorname{span}\left\{e_{1}, e_{2}\right\}$.

PRoOF. It follows from (3.4), (3.11) and (4.3) that

$$
\sum_{p}\left(h_{2 p}\right)^{2} X_{3}=0
$$

If $X_{3} \neq 0$, then obviously $h_{2 p}=0$. Then $X_{3}=0$, which together with (3.4), (3.11), (4.2) and $Y_{p i}=-\sum_{q} \phi_{p q} Z_{q i}$ implies that $h_{2 p}=0$.

LEMMA 4.2. Let $M$ be a real hypersurface in a complex space form $M_{n}(c), c \neq 0$, $n \geq 3$. If the structure Jacobi operator is cyclic-parallel, then the structure vector is principal.

PROOF. From Lemma 4.1 the matrix $\left(h_{p q}\right)$ is diagonalizable; that is, for a suitable choice of a orthonormal frame field $\left\{e_{p}\right\}$ we can set

$$
h_{p q}=\lambda_{p} \delta_{p q} .
$$

Here we shall set out some equations obtained from Lemma 4.1.

From (4.1), (4.2) and (3.13),

$$
X_{p}=Y_{p 3}=Z_{p 3}=Y_{p 2}=Z_{p 2}=0 .
$$

Equations (3.5), (3.6), (3.7) and (3.12) imply that

$$
\begin{gathered}
\left(\beta^{2}-\alpha \gamma\right)_{1}=0, \\
(\alpha \gamma)_{3}+\left(\beta^{2}-\alpha \gamma-c\right) X_{2}=0, \\
\left(\beta^{2}-\alpha \gamma-c\right)\left(X_{1}-\delta\right)=0, \\
\left(\beta^{2}-\alpha \gamma-c\right) X_{3}=0 .
\end{gathered}
$$

Put $p=q$ in (3.14). Then

$$
\left(\alpha \lambda_{p}\right)_{1}=0
$$

Moreover, from (112)-(32p),

$$
\begin{gathered}
\alpha_{2}-\beta_{1}=0 \\
\beta_{2}-\gamma_{1}=0 \\
(\alpha-\delta) \gamma-\beta X_{2}+(\gamma-\delta) X_{1}-\beta^{2}+c=0 \\
\alpha_{3}+3 \beta \delta-\alpha \beta+\beta X_{1}=0 \\
\beta_{3}+\gamma \delta+(\gamma-\delta) X_{1}-\beta^{2}=0 \\
\delta_{1}+\beta X_{3}=0 \\
\gamma_{3}-2 \beta \delta+(\gamma-\delta) X_{2}-\beta \gamma=0 \\
\delta_{2}+(\gamma-\delta) X_{3}=0 \\
\beta_{p}=0 \\
\gamma_{p}=0
\end{gathered}
$$




$$
\begin{gathered}
\alpha_{p}=\delta_{p}=0, \\
Y_{p 1}=Z_{p 1}=0 .
\end{gathered}
$$

It follows from $(q 1 p)$ and (3.14) that

$$
\alpha \beta Y_{q p}=\left(\alpha \lambda_{p} \lambda_{q}-\alpha^{2} \lambda_{p}+c \lambda_{p}-c \lambda_{q}-c \alpha\right) \phi_{p q}+\alpha_{1} \lambda_{p} \delta_{p q} .
$$

From this, $(2 p q)$ and $(q 3 p)$,

$$
\begin{aligned}
& {\left[\alpha \beta^{2}\left(\lambda_{p}+\lambda_{q}\right)-\left(\lambda_{p}-\gamma\right)\left\{\alpha \lambda_{p} \lambda_{q}-\alpha^{2} \lambda_{q}-c \alpha+c\left(\lambda_{p}-\lambda_{q}\right)\right\}\right.} \\
& \left.\quad-\left(\lambda_{q}-\gamma\right)\left\{\alpha \lambda_{p} \lambda_{q}-\alpha^{2} \lambda_{p}-c \alpha+c\left(\lambda_{q}-\lambda_{p}\right)\right\}\right] \phi_{p q}=0, \\
& \left(\lambda_{q}-\delta\right)\left[\alpha\left\{\left(\lambda_{q}\right)^{2}-\alpha \lambda_{q}-c\right\} \delta_{p q}+\alpha_{1} \lambda_{q} \phi_{p q}\right] \\
& \quad-\alpha \beta\left\{h_{q p 3}+\left(\lambda_{p}-\lambda_{q}\right) \Gamma_{q p 3}\right\}=0 .
\end{aligned}
$$

If $p=q$ in the above equation, then

$$
\left(\lambda_{p}-\delta\right)\left\{\left(\lambda_{p}\right)^{2}-\alpha \lambda_{p}-c\right\}-\beta\left(\lambda_{p}\right)_{3}=0 .
$$

In the following we shall abbreviate the expression 'take account of the coefficient of $\theta_{i}$ in the exterior derivative of ...' to 'see $\theta_{i}$ of $d$ of ...'.

Case I. Suppose that $\beta^{2}-\alpha \gamma-c \neq 0$. From (4.7) and (4.8),

$$
X_{3}=0, \quad X_{1}=\delta .
$$

It follows from (4.10), (4.15), (4.17), (4.5) and (4.11) that

$$
\alpha_{1}=\delta_{1}=\alpha_{2}=\delta_{2}=\beta_{1}=\beta_{2}=\gamma_{1}=0 .
$$

From (4.12), (4.13), (4.14) and (4.26),

$$
\begin{gathered}
\beta X_{2}+\left(\beta^{2}-\alpha \gamma-c\right)+\delta^{2}=0, \\
\alpha_{3}+4 \beta \delta-\alpha \beta=0, \\
\beta_{3}-\beta^{2}+2 \gamma \delta-\delta^{2}=0 .
\end{gathered}
$$

Seeing $\theta_{1} \wedge \theta_{3}$ of $d$ of $\theta_{23}$,

$$
\delta_{3}=-\beta \delta-2 X_{2} \delta,
$$

which, together with (4.28) and (4.29), implies that

$$
-2 \beta^{2} \delta+\alpha \delta^{2}+\alpha\left(\beta^{2}-\alpha \gamma-c\right)=0 .
$$

Seeing $\theta_{2}$ of $d$ of the above equation, we have that $\gamma_{2}=0$.

Now put $F=\alpha, \beta, \gamma$, and $i=1, j=2$ in Lemma 2.1. Then,

$$
\alpha_{3}(\gamma+\delta)=\beta_{3}(\gamma+\delta)=\gamma_{3}(\gamma+\delta)=0 .
$$

If $\gamma+\delta \neq 0$, then from (4.6) and (4.31) we have a contradiction. Thus $\gamma+\delta=0$, which also contradicts (4.6) and (4.31). 
Case II-1. Suppose that

$$
\begin{gathered}
\alpha_{1}=0, \\
\beta^{2}-\alpha \gamma-c=0 .
\end{gathered}
$$

Seeing $\theta_{2}$ of $d$ of (4.33),

$$
\left(\beta^{2}-\alpha \gamma\right)_{3}=2 \beta \beta_{3}-\gamma \alpha_{3}-\alpha \gamma_{3}=0 .
$$

From (4.12), (4.13, (4.14), (4.16) and (4.33),

$$
\begin{gathered}
-\delta \gamma-\beta X_{2}+(\gamma-\delta) X_{1}=0, \\
\alpha_{3}+3 \beta \delta-\alpha \beta+\beta X_{1}=0, \\
\beta_{3}+(\gamma-\delta) X_{1}+\gamma \delta-\alpha \gamma-c=0, \\
\gamma_{3}-2 \beta \delta+(\gamma-\delta) X_{2}+\beta \gamma=0 .
\end{gathered}
$$

On substituting (4.36), (4.37) and (4.38) into (4.34),

$$
(\delta-\gamma)\left(X_{1}-4 \alpha\right)=0,
$$

by virtue of (4.35). If $\delta=\gamma$, then by (4.33) we have a contradiction and hence

$$
X_{1}=4 \alpha .
$$

Substituting this equation into (4.35), (4.36) and (4.37),

$$
\begin{gathered}
\beta X_{2}=4 \alpha(\gamma-\delta)-\delta \gamma, \\
\alpha_{3}+3 \beta \delta+3 \alpha \beta=0, \\
\beta_{3}+3 \alpha \gamma-3 \alpha \delta+\gamma \delta=0 .
\end{gathered}
$$

It follows from (4.16), (4.33) and (4.40) that

$$
\alpha \gamma_{3}+\beta(3 \alpha \gamma-6 \alpha \delta-\gamma \delta)=0 .
$$

From (4.15) and (4.32) we have $X_{3}=0$ and therefore $\beta_{1}=\alpha_{2}=\delta_{2}=0$ because of (4.10) and (4.17). Hence, by (4.5), we have $\gamma_{1}=0$, and so $\beta_{2}=0$. From (3.6) we have $(\alpha \gamma)_{3}=0$. This, together with (4.33), implies that $\beta_{3}=0$. Therefore it follows from (4.18) that $\beta$ is constant.

Now put $F=\alpha$ and $\beta$ in Lemma 2.1. Then

$$
\alpha_{3}\left(\gamma+X_{2}\right)=0, \quad \beta_{3}\left(\gamma+X_{2}\right)=0 .
$$

If $\gamma+X_{2} \neq 0$, then $\alpha_{3}=\beta_{3}=0$. It follows from (4.20) that $\alpha$ and $\delta$ are constant. Furthermore, by (4.33) we see that $\gamma$ is constant. Thus from (4.41), (4.42) and (4.43),

$$
\begin{gathered}
\alpha+\delta=0 \\
3 \alpha \gamma-3 \alpha \delta+\gamma \delta=0 \\
3 \alpha \gamma-6 \alpha \delta-\gamma \delta=0
\end{gathered}
$$


Hence, by (3.4) and (4.33), $\alpha^{2}-c=0$ and $2 \beta^{2}+c=0$, which is a contradiction. Therefore $X_{1}=-\gamma$, which, together with (4.39), implies that $\gamma=-X_{1}=-4 \alpha$. Thus it follows from (4.41) that $\gamma_{3}=-4 \alpha_{3}=12 \beta(\delta+\alpha)$. Hence, from (4.43) we have a contradiction $\alpha \delta=0$.

Case II-2. Suppose that

$$
\beta^{2}-\alpha \gamma-c=0 \text { and } \alpha_{1} \neq 0 .
$$

Here we assert that if $\phi_{p q} \neq 0$, then $\lambda_{p}=\lambda_{q}$. To prove this, we assume that there exist indices $p$ and $q$ such that

$$
\phi_{p q} \neq 0, \quad \lambda_{p}-\lambda_{q} \neq 0 .
$$

Then from (4.23) and (4.44),

$$
\left(\lambda_{p} \lambda_{q}-2 c\right)\left(\lambda_{p}+\lambda_{q}\right)-2(\alpha+\gamma-\delta) \lambda_{p} \lambda_{q}-2 c \gamma-\delta\left\{\left(\lambda_{p}\right)^{2}+\left(\lambda_{q}\right)^{2}\right\}=0 .
$$

Multiply the above equation by $\alpha^{3}$ and see $\theta_{1}$ of $d$ of this equation. Then from (4.9),

$$
(\alpha \gamma)_{1}\left(\lambda_{p} \lambda_{q}-c\right)=2 \alpha_{1}\left(c \gamma-c \lambda_{p}-c \lambda_{q}-\alpha \lambda_{p} \lambda_{q}\right) .
$$

On the other hand, by (4.5), (4.10), (4.15) and (4.17),

$$
(\alpha \gamma)_{1}=2(\gamma-\delta) \alpha_{1},
$$

which, together with (4.46) and (4.47), implies that

$$
\lambda_{p} \lambda_{q}(\alpha+\gamma-\delta)=c\left(2 \gamma-\delta-\lambda_{p}-\lambda_{q}\right)
$$

Eliminate $(\alpha+\gamma-\delta) \lambda_{p} \lambda_{q}$ from this and (4.45):

$$
\left(\alpha \lambda_{p}\right)\left(\alpha \lambda_{q}\right)\left(\alpha \lambda_{p}+\alpha \lambda_{q}\right)-2 c \alpha^{2}(\alpha \gamma-\alpha \delta)-(\alpha \delta)\left\{\left(\alpha \lambda_{p}\right)^{2}+\left(\alpha \lambda_{q}\right)^{2}\right\}=0 .
$$

Multiply this equation by $\alpha^{3}$ and see $\theta_{1}$ of $d$ of this equation. Then by (4.9), $(\alpha \gamma)_{1}=-2(\gamma-\delta) \alpha_{1}$ and so $\gamma=\delta$ by virtue of (4.44) and (4.47). Thus from (3.4) and (4.43) we have contradiction. Therefore, for all $p, q$ such that $\phi_{p q} \neq 0$, we have $\lambda_{p}=\lambda_{q}$.

We now take $p, q$ such that $\phi_{p q} \neq 0$. Then, from (4.24) and $\lambda_{p}=\lambda_{q}$,

$$
\beta^{2} \lambda_{p}-\left(\lambda_{p}-\gamma\right)\left\{\left(\lambda_{p}\right)^{2}-\alpha \lambda_{p}-c\right\}=0 .
$$

Furthermore, from $(q 3 p),(4.9)$ and (4.22),

$$
\lambda_{p}\left(\lambda_{p}-\delta\right)=0 .
$$

Note that (4.25) implies that $\lambda_{p} \neq 0$. Hence we have $\lambda_{p}=\delta$. From (4.48) we have $(\alpha+\delta)(\delta-\gamma)=0$. If $\alpha+\delta=0$, then $\alpha$ and $\delta$ are constant, which contradicts (4.44). Hence $\delta-\gamma=0$. However, from (4.44) we have $\beta=0$, which is a contradiction.

Consequently we have proved $\beta=0$, which completes the proof. 


\section{Proof of Theorem 1.1}

In this section we prove Theorem 1.1. Let $M$ be a real hypersurface in a complex space form $M_{n}(c), c \neq 0, n \geq 3$. Suppose that the structure Jacobi operator is cyclicparallel. From $d \xi_{i}=0,(2.1)$ and (2.5),

$$
\Gamma_{1 i j}=\lambda_{j} \phi_{j i}
$$

If $\alpha=0$, then (3.2) implies that

$$
\left(\lambda_{i}-\lambda_{j}\right) \phi_{i j}=0 \quad \text { for } i, j \geq 2 \text {. }
$$

Thus $H$ and $\phi$ are commutative. By the classification theorems of real hypersurfaces in $M_{n}(c), c \neq 0$, due to Okumura [11] and Montiel and Romero [9], $M$ is locally congruent to one of the real hypersurfaces of type $A_{1}$ or $A_{2}$ in $P_{n} \mathbb{C}$ or of type $A_{0}, A_{1}$ or $A_{2}$ in $H_{n} \mathbb{C}$.

Suppose that $\alpha \neq 0$. We first prove that all principal curvatures are constant. From Lemma 4.1 and Proposition 2.3 we see that $\alpha$ is constant in $M$. We denote equation (3.2) by (32) $i j k$. By (213) and (313),

$$
\gamma_{1}=\delta_{1}=0
$$

It follows from $(32)_{i i i}$ and $(32)_{1 p p}$ that

$$
\left(\lambda_{p}\right)_{1}=\left(\lambda_{i}\right)_{i}=0 \quad \text { for } i \geq 2 .
$$

We take the indices $i$ and $j$ such that $i \neq 1, j \neq 1$ and $i \neq j$. Then eliminating $\left(\lambda_{i}-\lambda_{j}\right) \Gamma_{i j i}$ from $(i i j)$ and $(32)_{i i j}$, we have

$$
\left(\lambda_{i}\right)_{j}=0 .
$$

Hence, from (5.2), (5.3) and (5.4) all principal curvatures are constant. By the classification theorems of real hypersurfaces in $M_{n}(c), c \neq 0$, due to Kimura [7] and Berndt [1], $M$ is locally congruent to one of the homogeneous real hypersurfaces of type $A_{1} \sim E$ in $P_{n} \mathbb{C}$ or of type $A_{0} \sim B$ in $H_{n} \mathbb{C}$. So, we shall check equation (3.2) one by one for the above model spaces.

Here since all principal curvatures are constant, we shall rewrite the condition (3.2). For a suitable choice of a orthonormal frame field $\left\{e_{i}\right\}$ of each model space we set $h_{i j}=\lambda_{i} \delta_{i j}$. The Codazzi equation (2.3) asserts that

$$
\left(\lambda_{i}-\lambda_{j}\right) \Gamma_{i j k}=\left(\lambda_{k}-\lambda_{i}\right) \Gamma_{k i j}-c\left(\xi_{k} \phi_{i j}+2 \xi_{i} \phi_{k j}-\xi_{j} \phi_{i k}\right) .
$$

Therefore the cyclic-parallel structure Jacobi operator condition (3.2) is rewritten as

$$
\begin{aligned}
& 3 \alpha\left(\lambda_{i}-\lambda_{j}\right) \Gamma_{i j k}+3 \alpha c\left(\xi_{j} \phi_{k i}-\xi_{i} \phi_{j k}\right)-\left(\alpha^{2}+c\right)\left\{\delta_{i 1}\left(\lambda_{j}-\lambda_{k}\right) \phi_{j k}\right. \\
& \left.\quad+\delta_{j 1}\left(\lambda_{k}-\lambda_{i}\right) \phi_{k i}+\delta_{k 1}\left(\lambda_{i}-\lambda_{j}\right) \phi_{i j}\right\}=0 .
\end{aligned}
$$


Case I. $M_{n}(c)=H_{n} \mathbb{C}$. Let $M$ be of type $A_{0}$. Then $M$ has two distinct constant principal curvatures $\alpha=2$ and 1. Then it is easy to see that $M$ satisfies (5.6).

Let $M$ be of type $A_{1}$ or $A_{2}$. Then $M$ has three distinct constant principal curvatures $\sqrt{-c} / t, \sqrt{-c} t$ and $\alpha=\sqrt{-c}(t+1 / t)$, where $t=\tanh r$. For $i, j, k \geq 2$ we have (5.6) because the same principal curvatures exist in $\left\{\lambda_{i}, \lambda_{j}, \lambda_{k}\right\}$. Therefore, by (5.1) we obtain (5.6). For $j=1$ the left-hand side of (5.6) can be expressed as

$$
\left(\lambda_{i}-\lambda_{k}\right)\left(\alpha^{2} / 2-c\right) \phi_{i k}
$$

Since $2 \sqrt{-c} t-\alpha \neq 0$ and $2 \sqrt{-c} / t-\alpha \neq 0$, it follows from Proposition 2.4 that $\phi V_{\sqrt{-c} / t}=V_{\sqrt{-c} / t}$ and $\phi V_{\sqrt{-c} t}=V_{\sqrt{-c} t}$, where $V_{\lambda}$ denotes the eigenspace of $H$ with eigenvalue $\lambda$. Hence we have $\left(\lambda_{i}-\lambda_{j}\right) \phi_{i j}=0$ for any $i, j$. This, together with (5.7), implies (5.6). Thus the manifold $M$ satisfies (5.6).

Let $M$ be of type $B$. Put $j=1$ in (5.6). Thus by an argument similar to that above,

$$
\left(\lambda_{i}-\lambda_{k}\right)\left(\alpha^{2}-2 c\right) \phi_{i k}=0 .
$$

$M$ has three distinct constant principal curvatures $\sqrt{-c} / t, \sqrt{-c} t$ and $\alpha=$ $4 \sqrt{-c} t /\left(t^{2}+1\right)$, where $t=\tanh r$. Then, from Proposition 2.4, $\phi V_{1 / t}=V_{t}$ and therefore there exist indices $i$ and $j$ such that $\left(\lambda_{i}-\lambda_{j}\right) \phi_{i j} \neq 0$. This contradicts (5.8).

Case II. $M_{n}(c)=P_{n} \mathbb{C}$. Let $M$ be of type $A_{1}, A_{2}$ or $B$. By an argument similar to that in Case I, real hypersurfaces of type $A_{1}$ or $A_{2}$ satisfy the condition (5.6). Moreover if $\alpha^{2}=2 c$, then real hypersurfaces of type $B$ satisfy (5.6). The equation $\alpha^{2}=2 c$ tells us that $\cot r=(\sqrt{2 c+4}+\sqrt{2 c}) / 2$.

Let $M$ be of type $C, D$ or $E$. Then $M$ has five distinct constant principal curvatures. Put $j=1$ in (5.5) to get

$$
\left(\lambda_{k}-\lambda_{i}\right) \Gamma_{k i 1}=\left(\alpha \lambda_{k}-\lambda_{i} \lambda_{k}+c\right) \phi_{k i}
$$

by virtue of (5.1), which implies that

$$
2\left(\lambda_{k}-\lambda_{i}\right) \Gamma_{k i 1}=\alpha\left(\lambda_{k}-\lambda_{i}\right) \phi_{k i} .
$$

Suppose that $i, j, k \geq 2$ and $\lambda_{i} \neq \lambda_{j}$. Then it follows from (5.6) that $\Gamma_{i j k}=0$ and therefore $\theta_{i j}=\Gamma_{i j 1} \theta_{1}$. Seeing $\theta_{i} \wedge \theta_{j}$ of $d$ of $\theta_{i j}$,

$$
\lambda_{i} \lambda_{j}+c+\left(\phi_{i j}\right)^{2}\left\{\lambda_{i} \lambda_{j}+(\alpha / 2)\left(\lambda_{i}+\lambda_{j}\right)+3 c\right\}=0,
$$

because of (5.1) and (5.9). Put $i=1$ in (5.5). Then

$$
\left\{2 \lambda_{j} \lambda_{k}-\alpha\left(\lambda_{j}+\lambda_{k}\right)-2 c\right\} \phi_{j k}=0 \quad \text { for } j, k \geq 2 .
$$

From the above two equations it is easy to see that

$$
\lambda_{i} \lambda_{j}+c=0 \text { for } i, j \geq 2 \text { and } \lambda_{i} \neq \lambda_{j},
$$

which implies that $M$ has at most three constant principal curvatures. This is a contradiction.

This completes the proof of Theorem 1.1. 


\title{
References
}

[1] J. Berndt, 'Real hypersurfaces with constant principal curvatures in complex hyperbolic spaces', J. Reine Angew. Math. 395 (1989), 132-141.

[2] J. Berndt and H. Tamaru, 'Cohomogeneity one actions on noncompact symmetric spaces of rank one', Trans. Amer. Math. Soc. 359 (2007), 3425-3438.

[3] T. E. Cecil and P. J. Ryan, 'Focal sets and real hypersurfaces in complex projective space', Trans. Amer. Math. Soc. 269 (1982), 481-499.

[4] J. T. Cho and U.-H. Ki, 'Real hypersurfaces in complex projective spaces in terms of Jacobi operators', Acta Math. Hungar. 80 (1998), 155-167.

[5] U.-H. Ki, H. Kurihara and R. Takagi, 'Jacobi operators along the structure flow on real hypersurfaces in a nonflat complex space form', Tsukuba J. Math. 33 (2009), 39-56.

[6] U.-H. Ki and Y. J. Suh, 'On real hypersurfaces of a complex space form', Math. J. Okayama Univ. 32 (1990), 207-221.

[7] M. Kimura, 'Real hypersurfaces and complex submanifolds in complex projective space', Trans. Amer. Math. Soc. 296 (1986), 137-149.

[8] Y. Maeda, 'On real hypersurfaces in a complex projective space', J. Math. Soc. Japan 28 (1976), $529-540$.

[9] S. Montiel and A. Romero, 'On some real hypersurfaces of a complex hyperbolic space', Geom. Dedicata 20 (1986), 245-261.

[10] R. Niebergall and P. J. Ryan, 'Real hypersurfaces in complex space forms', in: Tight and Taut Submanifolds, (eds. T. E. Cecil and S. S. Chern) (Cambridge University Press, Cambridge, 1998), pp. 233-305.

[11] M. Okumura, 'On some real hypersurfaces of a complex projective space', Trans. Amer. Math. Soc. 212 (1975), 355-364.

[12] M. Ortega, J. D. Pérez and F. G. Santos, 'Non-existence of real hypersurfaces with parallel structure Jacobi operator in nonflat complex space forms', Rocky Mountain J. Math. 36 (2006), 1603-1613.

[13] J. D. Pérez, F. G. Santos and Y. J. Suh, 'Real hypersurfaces in complex projective spaces whose structure Jacobi operator is D-parallel', Bull. Belg. Math. Soc. Simon Stevin 13 (2006), 459-469.

[14] R. Takagi, 'On homogeneous real hypersurfaces in a complex projective space', Osaka J. Math. 19 (1973), 495-506.

[15] R. Takagi, 'Real hypersurfaces in a complex projective space with constant principal curvatures', J. Math. Soc. Japan 15 (1975), 43-53.

[16] R. Takagi, 'Real hypersurfaces in a complex projective space with constant principal curvatures II', J. Math. Soc. Japan 15 (1975), 507-516.

\author{
U-HANG KI, Department of Mathematics, Kyungpook National University, \\ Daegu 702-701, Korea \\ e-mail: uhangki2005@yahoo.co.kr
}

HIROYUKI KURIHARA, Department of Liberal Arts and Engineering Sciences, Hachinohe National College of Technology, Hachinohe, Aomori 039-1192, Japan e-mail: kurihara-g@hachinohe-ct.ac.jp 